PoS $\quad \begin{aligned} & \text { PROCEEDINGS } \\ & \text { OF SCIENCE }\end{aligned}$

\title{
First results from the NA62 straw spectrometer
}

\section{Nikolay Azorskiy}

Joint Institute for Nuclear Research

\section{Augusto Ceccucci}

European Organization for Nuclear Research

\section{Jerome Bendotti}

European Organization for Nuclear Research

\section{Hans Danielsson}

European Organization for Nuclear Research

Jordan Degrange

European Organization for Nuclear Research

\section{Neil Dixon}

European Organization for Nuclear Research

\section{Vladimir Elsha}

Joint Institute for Nuclear Research

\section{Temur Enik}

Joint Institute for Nuclear Research

\section{Levan Glonti}

Joint Institute for Nuclear Research

\section{Yury Gusakov}

Joint Institute for Nuclear Research

\section{Sergei Kakurin}

Joint Institute for Nuclear Research

\section{Vladimir Kekelidze}


Joint Institute for Nuclear Research

\section{Evgeny Kislov}

Joint Institute for Nuclear Research

\section{Alexander Kolesnikov}

Joint Institute for Nuclear Research

\section{Michal Koval}

Comenius University

\section{Peter Lichard}

European Organization for Nuclear Research

\section{Dmitry Madigozhin}

Joint Institute for Nuclear Research

\section{Joran Morant}

European Organization for Nuclear Research

\section{Sergei Movchan}

Joint Institute for Nuclear Research

\section{Francisco Perez Gomez}

European Organization for Nuclear Research

\section{Vito Palladino*}

CERN

E-mail: Vito.Palladinodcern.ch

\section{Irina Polenkevich}

Joint Institute for Nuclear Research

\section{Yuriy Potrebenikov}

Joint Institute for Nuclear Research

\section{Giuseppe Ruggiero}

European Organization for Nuclear Research

\section{Viacheslav Samsonov}




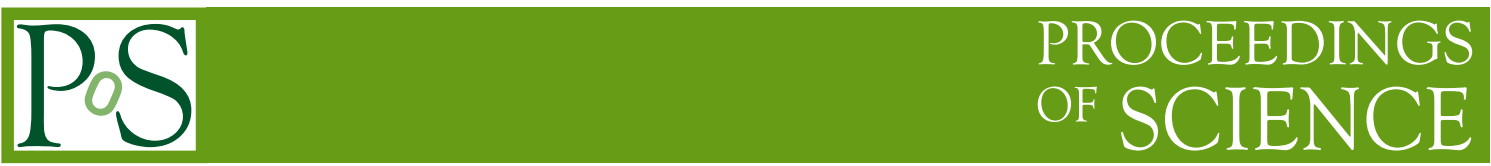

Joint Institute for Nuclear Research

\section{Sergey Shkarovskiy}

Joint Institute for Nuclear Research

\section{Alexander Sotnikov}

Joint Institute for Nuclear Research

The NA62 experiment at CERN is a fixed target experiment, it is located in the north area SPS high intensity facility. It aims at a precision measurement of the ultra-rare decay $K^{+} \rightarrow \pi^{+} v \bar{v}$. In order to achieve this goal a low mass $\left(1.8 \% X_{0}\right)$ spectrometer has been built to track charged kaon decay products. The system consists of 7000 straw tubes operating in vacuum. The analogic signals are shaped, amplified and discriminated by an ASIC chip (CARIOCA) mounted on the front-end board (cover) and a TDC was implemented in a FPGA. The data is sent from the cover to the Straw Readout Board (SRB) and then to the PC farm for analysis and storage. The first NA62 physics run took place in October-December 2014 and both the detector commissioning and the measured performance are presented. The goal of this presentation is to give a general overview of the system and in particular the readout scheme. The results obtained from the alignment, $\mathrm{r}-\mathrm{t}$ dependence, track fit and time resolution, are described in detail. A comparison with results from GARFIELD simulations will also be presented.

The European Physical Society Conference on High Energy Physics 22-29 July 2015

Vienna, Austria

* Speaker. 


\section{The NA62 experiment}

NA62 is a fixed target experiment at CERN [1], it aims at measuring the Branching Ratio (BR) of $\mathrm{K}^{+} \rightarrow \pi^{+} v \bar{v}$ predicted to be $\left(9.11 \pm 0.72 \times 10^{-11}\right.$ by the Standard Model (SM) [2]; such a rare process requires a significant technological challenge to achieve the needed background suppression $\left(O\left(10^{12}\right)\right)$, and to identify $\sim 100$ signal events within two years of data taking. The measurement of this branching ratio with an accuracy of $10 \%$ allows one to put constraints on several models concerning New Physics nevertheless a direct measurement of the CKM matrix element $\mathrm{V}_{t d}$.

NA62 has been designed to be an hermetic detector for ultra-rare kaon decays; this feature allows one to reduce efficiency problems of vetoes at very low energy and protects the measurement from low energy products of hadronic interactions. The apparatus consists of a long ( $\sim 250 \mathrm{~m}$ from target to dump) beam line. The detector can be ideally splitted in two groups of subdetectors. In in both sectors, particle identification and spectrometer detectors are foreseen, in order to identify and measure 4-momentum of particles. The beam related detectors are two, the first is a cedar detector called K-TAG able to identify the beam kaon component. Once identified a silicon spectrometer (GigaTraKer) measures the kaon 4-momentum. The second gorup contains sub-detectors dedicated to measure the $K^{+}$decay products. Particle identification and veto techniques are commonly adopted.

The experimental apparatus has been almost fully commissioned during a pilot run from midOctober to mid-December 2014. The first year of data taking started in June 2015.

A large fraction of the rejection power of the experiment is given by kinematic rejection, which relies on the measurements performed by the beam (GTK) and decay products (Straw Tracker) spectrometers. They are both designed to work in vacuum, but while the GTK is a small area silicon pixel detector, the latter is a $35 \mathrm{~m}$ long and $2 \mathrm{~m}$ wide straw based tracker. This last choice is determined by the need to minimize multiple scattering, which is the limiting factor in the achievable momentum resolution; thus the spectrometer has to be gas based and capable to operate in vacuum.

\section{The NA62 straw tracker design and electronics}

(spacers ancorati al frame di alluminio) The NA62 STRAW Spectrometer is composed of 4 chambers and 1 dipole magnet $\left(0.36 \mathrm{~T}, 270 \mathrm{MeV} / \mathrm{c} \mathrm{p}_{T}\right.$ kick). The chambers are separated by 10 $\mathrm{m}$, except for the last one $(15 \mathrm{~m})$, and the magnet is placed between the second and the third one. The spectrometer is integrated in the decay volume, a $100 \mathrm{~m}$ long and $2.5 \mathrm{~m}$ wide vacuum tube $\left(<10^{-6} \mathrm{mbar}\right)$, putting a tight constraint on the acceptable total leak rate $\left(<10^{-1} \mathrm{mbar} 1 / \mathrm{s}\right)$. Each chamber is composed of 4 views (X, Y, U and V), arranged in 2 modules (FIg. 1b), leaving a hexagonal hole of $\sim 12 \mathrm{~cm}$ diameter for the undecayed beam and with $2.1 \mathrm{~m}$ acceptance. Each view is composed by 4 layers of straws with a pattern that guarantees the geometrical coverage for tracks with an angle up to $\sim 50 \mathrm{mrad}$ from the normal to the view plane. The mechanical tolerances are quite tight, being $100 \mu \mathrm{m}$ for the straw straightness and $200 \mu \mathrm{m}$ for the position of the wire; this reflects also on the required quality of the aluminium frame, for which $100 \mu \mathrm{m}$ tolerance between the two farthest points has been achieved. This design should guarantee an 
average position resolution $\sigma<130 \mu \mathrm{m}$ for the individual view. The elementary active component is a drift tube $(\oslash=(9.75 \pm 0.05) \mathrm{mm}$ and $2.1 \mathrm{~m}$ long $)$ realized with a $36 \mu \mathrm{m}$ thick mylar wall straw, with $\mathrm{Cu}-\mathrm{Au}$ metallization $(50-20 \mathrm{~nm})$, produced by ultrasonic welding from a $\sim 3 \mathrm{~cm}$ wide tape, and a $30 \mu \mathrm{m}$ gold-plated tungsten wire. The straw is designed to be operated in vacuum, with an internal overpressure of $1 \mathrm{bar}$, and it is glued to the aluminium frame with a pretension of 1.5 $\mathrm{Kg}$; the combined effect of pretension and overpressure defines the shape of the straw, requiring additional constraints to obtain deviations less than $100 \mu \mathrm{m}$ with respect to the nominal position. This is achieved adding 2 very light spacers at $1 / 3$ and $2 / 3$ of the length. Few straws close to the not decayed beam passage have to be able to sustain a rate larger than $500 \mathrm{kHz}$. The anode and cathode resistance are $180 \Omega$ and $70 \Omega$ respectively. The tubes are grouped in cells of 16 for gas distribution and connection to the electronics. The front-end electronics is based on CARIOCA [3], and installed on a custom board (COVER) that is also part of the gas manifold. The signals from straw tubes are collected on one end by means of a flexible circuit (WEB), which hosts high voltage resistors and decoupling capacitors. Readout board (Straw Readout Board or SRB) is also a custom 9U VME board providing the control and data flow for 16 COVERs. The chosen gas mixture is $\mathrm{Ar}: \mathrm{CO}_{2}(70 \%: 30 \%)$, with an operating voltage of $1750 \mathrm{~V}$ (expected gain $10^{5}$ ).
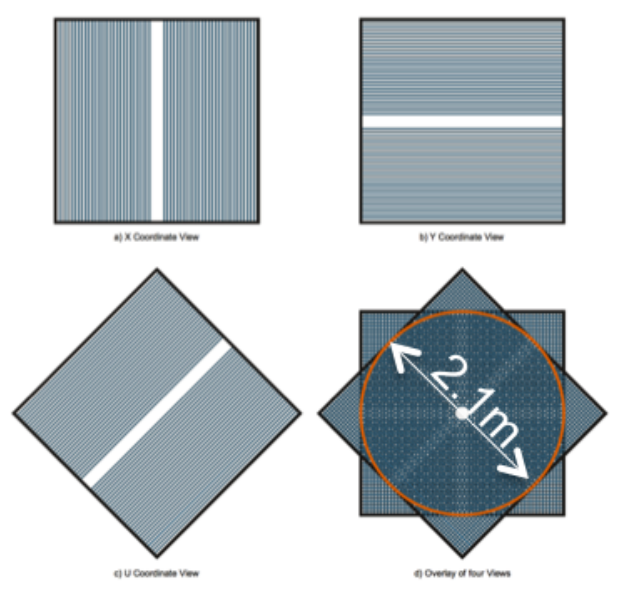

(a)

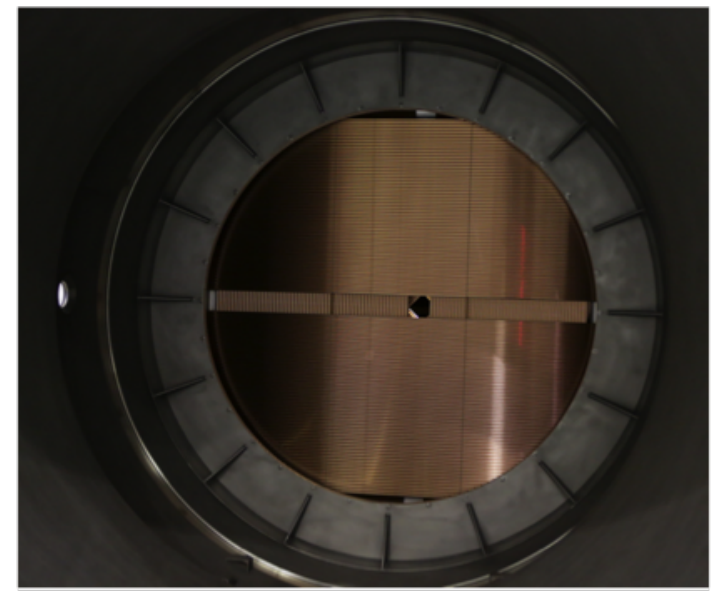

(b)

Figure 1: (a) Schematics of the views combination; (b) Final chamber installed on the beam line.

\section{Results from the NA62 pilot run}

The read-out system was triggerless and was tested up to the $30 \%$ of the nominal intensity ( $\sim 5 \mathrm{MHz}$ per chamber), limit imposed by the Gigabit Ethernet. The data shown in this section has been collected in the last ten days of the run with stable conditions. The reference detector used was the charged hodoscope that was also part of the trigger logic.

An on line monitoring system was implemented in order to monitor the detector performances. It was able to handle data from one full spill and plot it in an interactive ROOT GUI. Two very useful plots were the detector occupancy and spill time structure (PICTURES). These two plots 
were able to deliver important information concerning the status of the detector and beyond. In fact the straw detector was able to confirm $\mathrm{O}(\sim \mu \mathrm{s})$ fluctuations of the beam intensity (PICTURE).

A crucial step has been the detector calibration:

- Radius-Time curve ( $r-t$ curve)

- Time alignment

- Geometrical aliment

- Local magnetic field correction

As first approximation the $\mathrm{r}$-t curve has been computed using a GARFIELD simulation, no channel-by-channel fine tuning has been applied. An important upgrade will be the use of a muon run in order to calibrated the detector using an auto-calibration method [4].

Time alignment is performed channel-by-channel using the leading time cumulative distribution (Figure 2a) with a precision of $5 \mathrm{~ns}$.

Both geometrical alignment and magnetic field corrections have not been applied during the run.

The time resolution associated to the track has been computed as the mean value of the trailings of all the hits associated with the track during the reconstruction (Figure 4). The preliminary result is $\sim 6 \mathrm{~ns}$ that is within the expectations $(<10 \mathrm{~ns})$.

The missing square mass using the nominal beam direction has also been computed, the preliminary result is reported in Figure 3a. A major improvement of the resolution will follow the introduction of the measured beam particle track fit using the beam spectrometer (GTK).

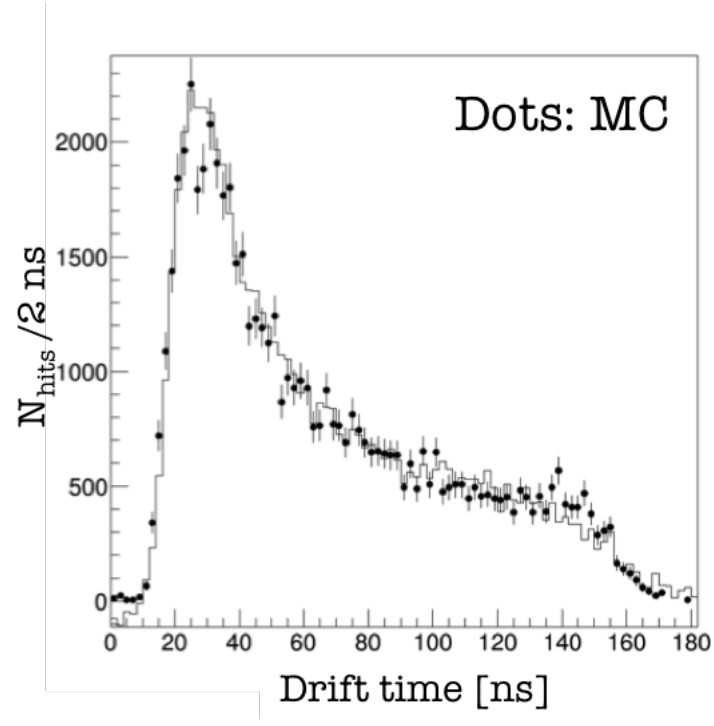

(a)

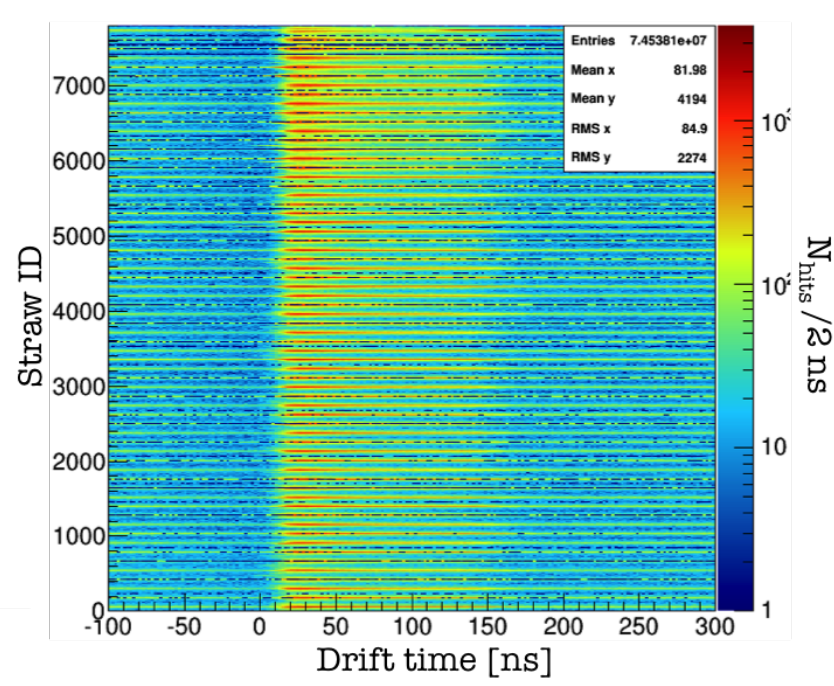

(b)

Figure 2: (a) Data-Montecarlo leading distribution comparison; (b) Leading distribution channelby-channel alignment. 


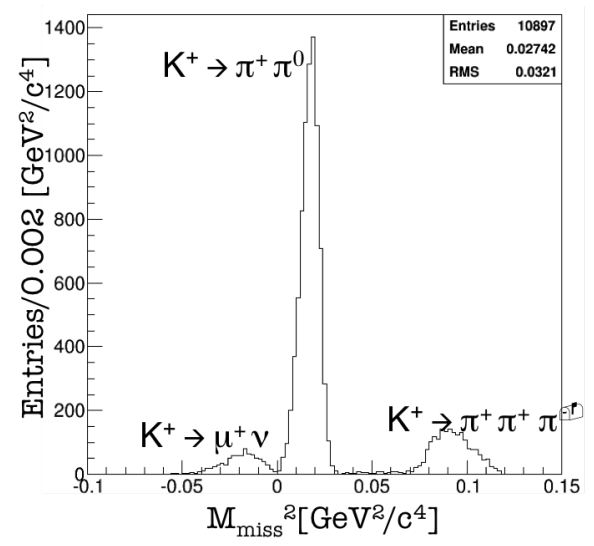

(a)

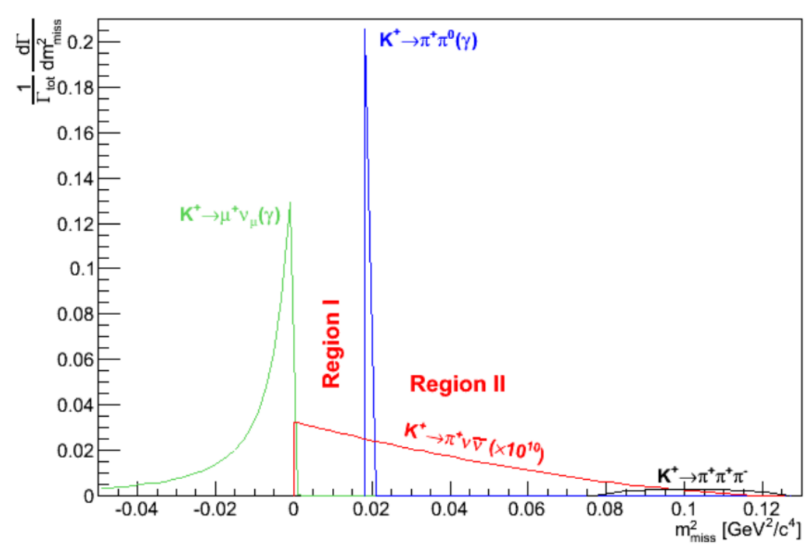

(b)

Figure 3: (a) Missing square mass for single pion reconstructed in the straws with a momentum below $35 \mathrm{GeV}$, the nominal beam direction has been used for the vertex reconstruction; (b) Analytical computation of theoretical behaviour of the missing square mass for the signal channel and the main backgrounds.

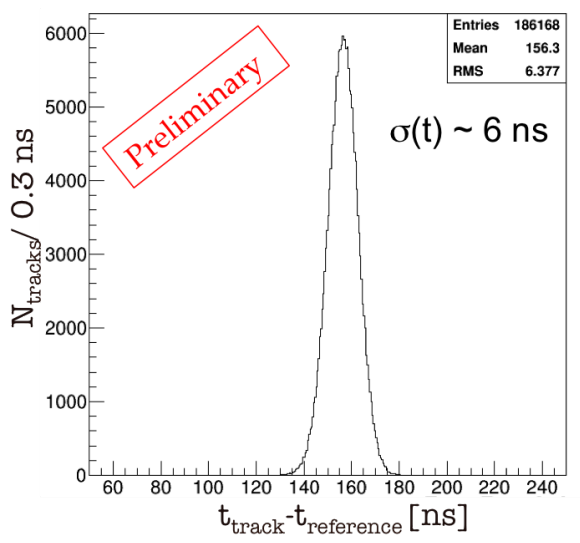

Figure 4: Time resolution of the trailings computed using hits belonging fro a reconstructed track.

\section{Conclusions}

The NA62 straw detector has been fully commissioned during the 2014 Pilot run (NovemberDecember).

The data collected were used to measure the detector performance. All the preliminary results are aligned with the expectations and further analysis are ongoing. In particular the next steps are the geometrical alignment, straw-by-straw calibration and local magnetic field corrections.

\section{References}

[1] G. Anelli et al., Proposal to Measure the Rare Decay $\mathrm{K}^{+} \rightarrow \pi^{+} v \bar{v}$ at the CERN SPS, 2005, CERN-SPSC-2005-013, SPSC-P-326. 
[2] A.J. Buras, D. Buttazzo, J. Girrbach-Noe and R.Knegjens, arXiv:1503.02693

[3] Moraes, D. and Bonivento, W. and Pelloux, Nicolas and Riegler, W., The CARIOCA Front End Chip for the LHCb muon chambers, 2003, LHCb-2003-009, CERN-LHCb-2003-009.

[4] Panda Collaboration, Technical Design Report for the: PANDA Straw Tube Tracker, arXiv:1205.5441. 